\title{
Propuesta de un modelo de gestión para mejorar la dirección municipal de La provincia de Otuzco
}

\begin{abstract}
RESUMEN
El presente artículo es un estudio de los órganos de gobierno y dirección del concejo municipal de otuzco, consistente en un análisis histórico y sociológico de sus funciones de gobierno, ejecutivo y técnico, en la perspectiva de mejorar la gestión municipal a través de la ejecución de obras de infraestructura y prestación de servicios públicos. Este empeño se complementó con un diagnóstico participativo municipal y encuestas a funcionarios del periodo 2007-2010. Los resultados obtenidos dan cuenta de desviaciones, diferencias y limitaciones en el cumplimiento de competencias, manejo de recursos y la prestación de servicios públicos, mostró asimismo la urgencia de transformar la administración pública para incrementar beneficios significativos y liderar el desarrollo local. Tras un análisis comparativo, se propone el "Modelo de Gestión EFQM" para alcanzar una buena gestión de procesos, personal, recursos, liderazgo del Gobierno y Órganos de Dirección, incidiendo en la calidad de vida de la ciudadanía; reduciendo carencias, pobreza extrema, mejorando competencias y desarrollo sostenido.
\end{abstract}

Palabras claves: desarrollo local, desarrollo sostenido y modelo de gestión efqm

"PROPOSAL OF A MANAGEMENT MODEL FOR IMPROVING MUNICIPAL ADDRESS OF THE PROVINCE OF OTUZCO"

\section{ABSTRACT}

The following article is a study of the government's structure and management of Otuzco Municipal's committee. This paper focuses on historical and sociological government function analyses, both executively and technically, with a purpose of purposing implementable improvements to the city's management of infrastructure and public service. The results from a survey collected between 2007 and 2010 showed areas of incompetence and limitations in the government's administration, use of resources and public service delivery. There is a need to implement public administration changes in order to increase the city's efficiency, improve standard of life for its citizens and further local development in certain key areas. After a comparative analysis, we propose the "Management Model EFQM.Therefore, the proposed "EFQM Management Model" provides suggestions to improve government leadership and structure effectively use resources and develop a realistic approach to reduce poverty and guarantee sustainable city development.

Keywords: local development, sustainable development and efam management model

\section{INTRODUCCIÓN}

En el Perú existe una compleja y rica tradición nativa, en la que florecieron más de un centenar de etnias con costumbres, lenguas y culturas distintas, cuyas organizaciones sociales, políticas, económicas y productivas se desarrollaron fuertemente condicionadas por una geografía compleja donde coexisten más de ochenta nichos ecológicos diferenciados, de los poco más de cien que se pueden catalogar en nuestro planeta. Este entramado de pueblos y culturas se vio sutil y violentamente sometido a un gobierno centralista, bajo una Corona distante y remota, a la que resultaba casi imposible acceder. Con un primer período, bajo la influencia de la Casa de Austria, fundamentalista y tiránica; y un segundo, bajo los Borbones, relativamente iluminado por las luces de una ilustración despótica; en este contexto hace poco menos de doscientos años nace la República como un estado unitario, dividido en regiones o departamentos, provincias y distritos.

Tras sucesivos intentos de regionalización, desde los esbozos teóricos que se formulan durante la dictadura del general Juan Velasco Alvarado, hasta el tímido impulso que cobra durante la presidencia de Alejandro Toledo Manrique, se configura el panorama actual, en el cual se atribuye a las municipalidades el rol de promotor del desarrollo local, un avance importante en términos conceptuales, si bien adolece de instrumentos y herramientas para cumplir la misión. La legislación concede al municipio autonomía política, por lo cual emite normas con categoría de ley material. Autonomía local, con la facultad para promulgar Ordenanzas con el fin de lograr el desarrollo local. Autonomía Administrativa, que lo faculta para emitir reglamentos en actos administrativos de contratación y ejecución de las decisiones y autonomía económica, para disponer y generar sus recursos y decidir sobre su presupuesto.

El gobierno militar (1968-1980) liquidó los procesos electorales y se facultó a nombrar alcaldes, lo cual se mantuvo hasta el segundo gobierno del arquitecto Fernando Belaunde Terry. Sin un modelo de municipio como eje del desarrollo local, se generaron experiencias de gestión replicables fácilmente: participación ciudadana, protección del medio ambiente, gestión participativa, políticas sociales y asociatividad municipal. No obstante, estas experiencias no se masificaron ni se convirtieron en política na-

1 Master en Gestión Industrial, profesor de Gestión Estratégica en la Escuela de Postgrado de la Universidad Nacional de Trujillo, e-mail: wehraqo@hotmail.com. 
cional, sino que fueron referentes de cambios legislativos, desdeñados por los actores políticos e interrumpidos por el gobierno autocrático de Alberto Fujimori Fujimori.

Durante la década de los años noventa se agudizó la dependencia hacia el gobierno central, lo cual propició el clientelismo del régimen, ello limitó la asociación y autonomía municipal, especialmente en los ámbitos provinciales. Se incrementó el gasto y la autonomía de los municipios distritales, y se aseguró así el objetivo político de evitar el desarrollo de liderazgos opositores mediante el Fondo de Compensación Municipal, como mecanismo de descentralización fiscal que, sin embargo, debe protegerse y potenciarse.

El impulso a la descentralización del 2002 y la reforma del ordenamiento legal de la organización municipal en los órganos de gobierno y dirección (3), recoge lo aprendido en las experiencias locales más avanzadas. Lejos ya de los primeros cabildos que fundaron los conquistadores ibéricos, la breve síntesis del último medio siglo sirve para comprender mejor el punto en que nos encontramos; con un municipio más próximo al ciudadano, donde el Gobierno Local está llamado a gestionar los programas sociales. No obstante, existe una evidente falta de voluntad política para descentralizar estos programas; por una parte, dada su rentabilidad política, y por otra, debido a la tradicional desconfianza en los organismos "provincianos". Por ejemplo, las municipalidades provinciales han respondido en los programas de complementación alimentaria transferidos con limitaciones debidas al desabastecimiento, y con los parches de intervenciones de emergencia. Las transferencias de programas sociales son insuficientes, los programas que fueron cuestionados se excluyeron del proceso: el Programa nacional de manejo de cuencas hidrográficas y conservación de suelos, Pronamachcs, pese a estar incluido en el Plan Anual de Transferencias 2003. La transferencia del Instituto Nacional de Infraestructura Educativa, Infes, no ha sido incluida en el proceso a la fecha. Con el objetivo de promover la compra de productos locales, los comités de gestión de los programas transferidos tienen la responsabilidad de elaborar las canastas alimentarias de cada provincia, pero las discrepancias generan desviaciones.

\section{Los Recursos de los gobiernos locales}

Los recursos económicos se han venido incrementando de manera notable, como resultado del crecimiento sostenido de la economía del país y con la creación de nuevos cánones, sobrecánones y regalías, así como por el aumento de cantidad y valor de los minerales, del gas y el petróleo. Por otro lado, las que cuentan con una base demográfica y productiva de relativa importancia pueden generar recursos propios significativos, a diferencia de las más pequeñas, rurales y pobres, que dependen del FONCOMÚN y por último, están las que se encuentran en zonas mineras y petroleras, pues los recursos del canon, se han convertido en una importante fuente de financiamiento.

La inadecuada ejecución del gasto municipal y el aumento del impuesto de promoción municipal, que se distribuye a través del FONCOMÚN, han generalizado las críticas relativamente fundadas, por lo que es necesario la creación del Fondo de Estabilización del Canon, el cual evitaría que se tenga que gastar los recursos bajo presión o sin tener realmente una buena cartera de proyectos, permitiendo asegurar los recursos para épocas en las que caiga el precio de los minerales; sin embargo, subsiste una mala calidad de gasto que podría enfrentarse desde estrategias de desarrollo de capacidades y/o esfuerzos de asociativismo municipal para la preparación de carteras de proyectos, lo cual contribuiría al desarrollo de la localidad de manera mucho más eficiente.

La gestión municipal, no solo se circula con el aspecto de servicios, sino que también, implica diseñar políticas públicas locales y establecer políticas sociales de educación, salud, nutrición, etc, como también promover el desarrollo económico, ambiental e institucional en beneficio de los ciudadanos (1). La mayoría de municipalidades siguen el mismo organigrama de gobierno y dirección con patrón tradicional; por lo que, entender al Gobierno Local implica tener otro espejo con una visión mucho más amplia, cual organice, reorganice, expanda o contrae; para diseñar políticas locales que busquen en todo momento producir valor público, mejorando las condiciones y la calidad de vida de los ciudadanos.

Los alcaldes y conductores de una determinada municipalidad disponen de cuatro años para conducir la gestión municipal, pues siendo los máximos representantes del Estado subnacional, actuarán o se desenvolverán durante su periodo de gestión en torno a su propuesta de proyecto o programa de campaña electoral, orientado a brindar eficiencia y transparencia de gestión; esto quiere decir, encausarse en un prototipo multiorganizacional y multisectorial de gobierno municipal compuesto de tres partes interactuantes: gestión municipal integral, de servicios y gestión de resultados (8). 
La municipalidad, en su función promotora local de desarrollo, debe actuar como entidad integradora y planificadora estratégica; además, debe ser receptiva y comprensible dentro de una cultura organizacional integral y accesible, lo cual implica una comunicación eficiente con la ciudadanía y una postura sensible a las necesidades en la medida en que está llamada a atender las demandas de la población, como también actuar de prestadora local fomentando la participación activa de la ciudadanía organizada (1). Algunos Municipios adolecen de falta de planificación propia y deben asumir, no obstante, el reto de continuar bajo parámetros de la gestión saliente; por lo tanto, continúan el curso dejado. En otros casos, actuarían en forma desordenada, sin contar con una visión de desarrollo, este factor recurrente es precisamente uno de los argumentos del Centralismo. La gestión municipal debe encajar tanto con la gestión regional como con la gestión nacional, en el marco de los acuerdos regionales; es decir, en el Plan de Desarrollo Concertado Regional, en el que se encuadra el Plan de Desarrollo Municipal, el Plan Operativo Institucional, entre otros. Esto daría un quiebre y corte al modelo tradicional de gestión que se ha venido operando desde hace más de tres décadas, o sencillamente, mantendría el modelo de gestión que sigue vigente y que actualmente se encuentra en crisis, esta la disyuntiva que debemos resolver.

\section{Configurar la gestión de los servicios municipa- les}

Los modelos (8) constituyen una aproximación en la construcción de una base teórica conceptual y operativa para diseñar una Hoja de ruta que permita la implementación de la visión, las metas y objetivos del Plan Estratégico y el Plan de Desarrollo Concertado del gobierno local, donde la sociedad civil se involucre analizando la productividad, fiscalizando idóneamente, optimizando el uso de recursos hacia una con inclusión sostenible, visionando el futuro que se desea del municipio. Los elementos centrales están referidos al tratamiento del desarrollo de la gestión municipal, desde la perspectiva de los órganos de gobierno y de dirección, para realizar cambios en la concepción, visión y acción a favor del desarrollo integral de la comunidad excluida. La factibilidad del modelo adecuado se obtuvo de un análisis metodológico comparativo de los vigentes en trabajos interrelacionados y sensibles a la sociedad civil, con el objetivo de monitorear los elementos que contribuyen a superar las mayores carencias de la gestión, incrementando eficiencia en el municipio.
Por otra parte, hay otros elementos que contribuyen a superar las carencias de la gestión, incrementando la eficiencia en el municipio.

El análisis se practicó sobre esquemas técnicos identificados en base a variables definitorias: misión, enfoque, esquema estructural, sistema de retroalimentación o feedback, criterios y subcriterios, además de los conceptos o principios fundamentales (2):

De los distintos modelos que buscan la excelencia de la gestión empresarial, se han comparado sistemáticamente cuatro de ellos:

Modelo de Malcolm Baldrige (10) el cual se basa en un sistema de liderazgo, planificación estratégica y enfoque hacia el cliente y el mercado.

Modelo Gerencial Deming (4), este se sustenta en crear un sistema organizativo para la cooperación interna y externa que fomente el aprendizaje y la implementación en prácticas de gestión de procesos; llevando satisfacción al personal, fundamentalmente para la satisfacción del cliente y la supervivencia de la organización.

Modelo Iberoamericano (6), es muy similar al EFQM debido a que los resultados excelentes se consiguen con liderazgo, estilo de dirección y procesos adecuados.

Modelo EFQM (5), basa los resultados excelentes en el rendimiento, los clientes, el personal y la sociedad, como impacto de la política y la estrategia del liderazgo.

El enfoque: tanto el modelo EFQM como el modelo Malcolm Baldrige tienen el mismo objetivo al establecer un conjunto de criterios e impulsar la aplicación de estos, como si se tratase de una herramienta de autoevaluación.

El esquema estructural: El modelo EFQM y el iberoamericano tienen mucho en común. Debido a que el segundo está basado en el primero, comparten un esquema similar y existen pocas diferencias.

Sistema de retroalimentación: Cabe destacar las diferencias entre ellos, pero todos estos están en la misma dirección que estiman oportuno las necesidades de la organización.

Comparativa de criterios y subcriterios. El modelo EFQM tiene 9 criterios, los cuales se subdividen en 32 subcriterios. Mientras que, el modelo Iberoamericano cuenta con 9 criterios que se subdividen en 28. El modelo Malcolm Baldrige posee 7 criterios, los que se subdividen en 19 subcriterios y, por último, el modelo Deming no contiene ninguno. 
A diferencia de los otros modelos, el EFQM y el lberoamericano resultan más apropiados para la gestión pública en la medida en que tienen un carácter ético únicamente técnico.

\section{MATERIAL Y MÉTODOS}

\subsection{Objeto de estudio}

El mandato del alcalde y regidores está sujeto a reglas de gobierno, soslayarlo desafía la legitimidad y genera un resquebrajamiento de la confianza por parte de la población. Por lo que resultan resaltantes los pedidos de 186 solicitudes de revocatoria aceptadas por la ONPE (el número podría aumentar). Las solicitudes tramitadas constituyen el 8,5\% de los 2.179 kíts adquiridos en la institución electoral hasta abril 2012; 30 pertenecen a La Libertad en las jurisdicciones de la provincia de Bolívar, Ascope, Julcán, Pataz, Santiago de Chuco, Pacasmayo, Chepén, Trujillo y Otuzco. -nueve de las solicitudes de revocatoria son distritales-. El trabajo, abordó específicamente la gestión gerencial de la municipalidad; es decir, a los órganos de gobierno y dirección con el propósito de comprender la labor efectuada para incidir eficientemente en su razón de ser o finalidad señalada en la Ley Orgánica de municipalidades.
El presente trabajo se realizó en la capital de la provincia de Otuzco, con $448.57 \mathrm{Kms}^{2}$ de superficie y una población de 97,056 habitantes; 48,222 hombres y 47,784 mujeres, densidad poblacional de 53,65 habitantes por $\mathrm{Km}^{2}$. $62.20 \%$ de población rural y una tasa de crecimiento de $1,6 \%$ para el año $2010^{2}$. La población urbana asciende a 20,460 habitantes, la rural a 75,546 habitantes con 03 cuencas hidrográficas y 1,740 Unidades Agropecuarias comprendidas en 11 distritos; en la cuenca del río Moche se encuentran Otuzco, Salpo, Mache y Agallpampa, en la cuenca del río Chicama, Charat, Huaranchal y Usquil ${ }^{3}$. En la cuenca del río Sinsícap están La Cuesta, Paranday y Sinsícap.

Uno de los principales problemas que enfrenta el distrito de Otuzco es el mal estado en el que se encuentran sus vías, tanto las urbanas como los caminos vecinales que conectan la zona urbana con los centros poblados rurales, esto origina un elevado costo de operación vehicular, e insatisfacción generalizada de la población por las deficiencias del servicio. Por otra parte, la infraestructura social y productiva existente continúa muy deteriorada, debido a las constantes lluvias intensas y al exponerse a un incremento de tráfico rural por estas vías; necesitándose por lo tanto un mejoramiento y ampliación de las vías que circundan a la provincia de Otuzco.

Tabla N. $^{\circ}$ 1. Cifras relacionadas con el Índice de Desarrollo Humano ${ }^{4}$

Población sin acceso al agua potable $32 \%$

Población sin acceso a desagüe y letrinas $50 \%$

Población sin acceso a energía eléctrica $60 \%$

Población masculina analfabeta 6,214 7.0\%

Población femenina analfabeta 10,831 12.2\%
El Índice de Desarrollo Humano (IDH) ${ }^{5}$. Pues bien, para La Libertad el IDH se coloca en un pobre 0.6046 , mientras que, en Otuzco apenas alcanza 0.5583.

Ordenada la población nacional según el ingreso per cápita (INEI), Otuzco se encuentra en el quintil61, el de menores ingresos.

Tabla N. ${ }^{\circ} 2$ Recursos Humanos que atienden las demandas de salud ${ }^{7}$

Médicos 0.62

Enfermeras 0.78

Obstetrices 0.18

Odontólogos 0.03

Asistentas sociales 0.03

Químicos farmacéuticos 0.06

Nutricionistas 0.12

Fisioterapistas 0.06

Laboratoristas clínicos 0.09

En la actualidad más de los $80 \%$ de las afecciones de salud diagnosticados en la Provincia de Otuzco se deben a dos grupos de enfermedades: las infecciosas y las deficiencias nutricionales. En ambos casos las dolencias tienen relación con la carencia de agua potable y desagüe.

\section{INEI, Encuesta ENHAO 2009.}

Dirección de Agricultura La Libertad.

Se define el quintil como la quinta parte de una población estadística ordenada de menor a mayor en alguna característica.

Concepto elaborado por el Programa de las Naciones Unidas para el Desarrollo (PNUD). Este se basa en un indicador social estadístico, compuesto por tres parámetros: vida larga y saludable, educación y nivel de vida dignos; donde el máximo ideal es la unidad.

6 Se define el quintil como la quinta parte de una población estadística ordenada de menor a mayor en alguna característica.

7 Porcada mil habitantes, Minsa 2006 


\section{Componentes de Evaluación:}

Prestar servicios públicos municipales, ejecutar obras de infraestructura local para satisfacer las crecientes necesidades de la población otuzcana requiere aprobar y hacer seguimiento del cumplimiento de sus competencias atribuidas por la Constitución Política y desarrolladas en la Ley de Municipalidades en el marco de la normatividad vigente del ámbito de la descentralización nacional. El estudio del gobierno local consideró los siguientes componentes de evaluación: los órganos de gobierno y dirección (3), que deciden libremente determinados asuntos de orden legal y administrativo. Los Instrumentos de Gestión (3), Plan de Desarrollo Municipal Concertado, Presupuesto Participativo, Plan de Desarrollo Institucional, Plan de Acondicionamiento Territorial, Plan de Desarrollo Urbano, Plan de Desarrollo Rural y el Sistema de Gestión Ambiental Local. El Marco Organizativo, acreditado públicamente como organización municipal. Los Órganos de Dirección de línea y apoyo, conformados por las gerencias municipales: administración, asesoría jurídica, servicios públicos, desarrollo urbano, desarrollo social, rentas, procuraduría pública y Los órganos de asesoramiento. Por otra parte, las acciones enmarcadas en el Reglamento del Concejo Municipal, el Presupuesto Anual y sus modificatorias, el Cuadro de Asignación de Personal y el régimen de organización interior de la municipalidad (ROF-MOF-MAPRO-TUPA).

El Distrito de Otuzco cuenta con una población de 27,134 habitantes (Proyección al 2010, INEI, 1999). Dividido en cinco sectores: San Venancio, San Remigio, San Agustín, El Olivo y Walter Acevedo. Asimismo, se identifican 7 barrios: La Ermita, Central, Cruz Blanca, La Retama, Ramón Castilla, San Antonio y Santa Rosa. Para optimizar la gestión se realizó el estudio definiendo al municipio como universo y población muestra aleatoria simple de 50 trabajadores de la dirección y gestión. Variable Independiente: el modelo de gestión y variable dependiente: la dirección municipal.

Prioridades a resolver por la municipalidad al 2014. Se identificó y jerarquizó los problemas por sectores: salud, educación, fomento y promoción del empleo, agricultura, ganadería, turismo, infraestructura vial, medio ambiente, seguridad ciudadana, participación y vigilancia ciudadana, y desarrollo humano integral; por lo expuesto, se considera pertinente la propuesta de un modelo de gestión para mejorar la Dirección del Concejo Provincial de Otuzco.

\subsection{Análisis de los medios de gestión}

Hacer sustentable la capacidad de representación como promotora del desarrollo integral sostenible y prestadora de servicios, requiere de datos archivados en los reportes, informes finales de gestión de la dirección de los órganos de gobierno y dirección, específicamente requiere de la Gerencia General (8). Las rentas vigentes, la capacidad de gasto, la tributación municipal, los recursos humanos; así como los Planes de Desarrollo Urbano y Rural, los Proyectos y el Presupuesto de la Gerencia de Planificación, sumados a la explicación de ordenanzas, los lineamientos, normativas, reglamentos o actos administrativos generados e implementados internamente y/o emitidos en el ámbito de los grados de libertad en Gerencia Jurídica, constituyen en conjunto los medios de gestión de mayor importancia en nuestro estudio, acotado entre los años 2007 hasta el 2010.

A las gestiones propias del Municipio, se debe sumar el reconocimiento de iniciativas de valor agregado, las inversiones, la capacidad de gestión independiente; así como la capacidad de decidir y ordenar en las funciones y competencias exclusivas facultadas por ley para impulsar el desarrollo local y replicables en otra entidad de gobierno local desarrolladas por la sociedad civil. Se realizó encuestas formuladas a medida, en una población encuestada de 200 personas: 30 docentes de la UGEL, 20 profesionales del sector salud, 30 comerciantes, 30 dirigentes vecinales y 10 deportivos, 20 pequeños empresarios agrícolas y 10 mineros. Por último, se seleccionó 50 funcionarios ediles del personal de la Municipalidad. Esto permitirá reconocer la existencia y tendencia de desviaciones y/o diferencias en el cumplimiento de su finalidad.

\subsection{Métodos y técnicas}

Hemos elaborado un diagnóstico socioeconómico de la provincia, enmarcado en el contexto del Municipio. Para el análisis social, se realizó un estudio dinámico de la población con relación a las principales variables e indicadores demográficos. La estructura y composición (población por rangos de edad, sexo, etc.) se realizó con un análisis comparativo intercensal entre los años 1981-1993.

La caracterización social de las variables que describen las Necesidades Básicas Insatisfechas (NBI), se realizó a partir de la información de resultado del IX Censo Nacional de Población y Vivienda. Aplicamos el método deductivo-inductivo e histórico social, según lo requiriera el caso. Mediante 
la estadística se identificaron los hechos para su análisis cuantitativo y cualitativo; que finalmente son procesados mediante el método analítico-sintético. Complementariamente, se aplicaron encuestas (entrevista-cuestionario) para el diagnóstico del grado de pobreza, capacidad competitiva, entorno económico, tipos de competencia de la finalidad del municipio en el proceso de descentralización y vinculando los análisis a los objetivos del estudio:

Método deductivo (9). Se empleó para identificar causas de finalidad de representación y desarrollo sostenible municipal en las revocatorias de alcaldes, regidores y conflictos medioambientales específicos, especialmente en la adecuación de la LOM por los órganos de gobierno y dirección de la municipalidad provincial de Otuzco.

Método inductivo (9). Facilitó organizar la información y documentación obtenida de la muestra para formular los cuestionarios y entrevistas. El contraste con lo general sirvió para focalizar la problemática y disponer respuestas a priori. El trazado inductivo de la autonomía municipal de Otuzco conduce a replantear ajustes al modelo de gestión.
Método analítico (9). Se evaluaron en forma desagregada los instrumentos de gestión para identificar los criterios y prioridades del Órgano de Gobierno y Dirección. En los instrumentos de recojo de información como entrevistas, cuestionarios de las encuestas e informes se consideraron un tamizado comparativo a fin de certificar las distorsiones y diferencias con respecto a lo que serían los resultados satisfactorios.

El diseño de investigación es de una sola casilla y no experimental transeccional de tipo descriptivo. Este diseño muestral es del tipo no probabilístico, se determina una cantidad a encuestar, los funcionarios fueron entrevistados de acuerdo a la muestra representativa seleccionada con la técnica del muestreo aleatorio simple estratificado. Aplicamos una muestra estratificada por niveles para seleccionar a los participantes, siguiendo el método del azar simple, de acuerdo a la estadística del 10\%, representativo en poblaciones menores, utilizado por Rochaerfield, citado por Tecla, A. 1978: Métodos y técnicas de investigación en ciencias sociales.

\section{Resultados}

Tabla N. ${ }^{\circ}$ 3. Situación: Órgano de Gobierno y Dirección

\begin{tabular}{|c|c|}
\hline $\begin{array}{l}\text { Órganos de gobierno } \\
\text { y dirección }\end{array}$ & Documentación y entrevistas \\
\hline Gestión institucional & $\begin{array}{l}\text { El } 80 \% \text { de los informes de hallazgo de contraloría recomienda a los órganos de apoyo y línea un } \\
\text { mayor celo en el marco normativo y técnico por parte de autoridades y funcionarios. } \\
\text { El } 60 \% \text { de los planes concertados y presupuesto participativo son ajustados al protocolo de ley en el } \\
\text { gabinete y carecen de vigilancia ciudadana. } \\
\text { El } 60 \% \text { de las transferencias recibidas están cuestionadas por la comunidad de Otuzco/municipalidad } \\
\text { Otuzco (S/. Nuevos soles): Acreditación MEF } \\
2007: 50,077,253.01 \text { / Municipalidad de Otuzco } 11,587,823.37 \\
2008: 48,341,163.91 / \text { Municipalidad de Otuzco } 11,717,218.76 \\
2009: 68,201,187.02 \text { / Municipalidad de Otuzco } 15,882,443.02 \\
2010: 78,706,649.37 / \text { Municipalidad de Otuzco } 19,229,050.27 \\
2011: 94,281,064.23 / \text { Municipalidad de Otuzco } 24,679,595.43 \\
\text { Plan de Desarrollo Municipal no existe. } \\
30 \% \text { funcionarios ediles denunciados por corrupción por la población y } 70 \% \text { de quejas registradas por } \\
\text { la ciudanía no atendidas durante los últimos años. } \\
\text { Solo el } 5 \% \text { de reclamos y quejas de área son atendidos por comisiones de regidurías. } \\
\text { El } 100 \% \text { de los funcionarios coinciden en la necesidad de realizar un plan de capacitación gestión } \\
\text { institucional (Fig. } 1 \text { ) }\end{array}$ \\
\hline $\begin{array}{l}\text { Desarrollo } \\
\text { social }\end{array}$ & $\begin{array}{l}\text { El } 90 \% \text { de las recomendaciones de auditoría a los servicios sociales en planificación, inequidad de } \\
\text { inversión en la extrema pobreza y desatención de grupos vulnerables y escasa promoción de la par- } \\
\text { ticipación ciudadana no son implementados. } \\
\text { El } 70 \% \text { de la demanda de servicios de salud es en medicina interna, particularmente en las poblacio- } \\
\text { nes más alejadas de la capital. } \\
\text { No existe inversión en infraestructura para la cultura y el deporte. } \\
\text { Un } 90 \% \text { manifestaron que resulta necesario mejorar la gestión de desarrollo social, por cuanto es la } \\
\text { política actual del gobierno central respecto a la inclusión social (Fig. } 2 \text { ). }\end{array}$ \\
\hline
\end{tabular}




\begin{tabular}{|c|c|c|}
\hline & \multicolumn{2}{|c|}{$\begin{array}{l}\text { Desordenado crecimiento urbano no planificado, ausencia de catastro y plan de expansión urbana } \\
\text { a nivel provincial y distrital, agudizando las carencias de los servicios básicos y de infraestructura } \\
\text { sanitaria. } \\
\text { No existen Plan de desarrollo vial interno, los medios de transporte terrestre no acceden a los case- } \\
\text { ríos y anexos, Plan de Desarrollo Urbano y Plan de Desarrollo Rural. } \\
\text { El } 60 \% \text { de los funcionarios opinaron que los Planes de desarrollo vial y urbano, resultan necesarios } \\
\text { diseñarlos, manifestando además la necesidad de crear circuitos turísticos. fig. } 3\end{array}$} \\
\hline Seguridad ciudadana & \multicolumn{2}{|c|}{$\begin{array}{l}\text { EL } 80 \% \text { de denuncias registradas en el ámbito urbano es por seguridad pública y el } 90 \% \text { de denun- } \\
\text { cias en área rural es por propiedad de tierras. } \\
\text { Ausencia de equipos digitales para el sistema provincial de Seguridad Ciudadana. } \\
\text { Un } 80 \% \text { de los funcionarios advierte la ausencia de una política adecuada con respecto a la proble- } \\
\text { mática de seguridad ciudadana, lo cual es un tema de relevancia actual (Fig. 4). }\end{array}$} \\
\hline & \multicolumn{2}{|c|}{$\begin{array}{l}0 \% \text { de ordenanzas para combatir la contaminación por minería informal, relaves mineros y uso indis- } \\
\text { criminado de agroquímicos por las cercanías a fuentes naturales de agua y suelos. } \\
50 \% \text { de los entrevistados se muestran a favor de un Plan Estratégico de Gestión Ambiental, a pesar } \\
\text { de los evidentes problemas ambientales y el perjuicio que generan en el agro (Fig. } 5 \text { ) } \\
\text { No existe Plan de acondicionamiento territorial y Sistema de gestión ambiental local. } \\
\text { Autoridades e instituciones desconocen las potencialidades ecológicas de la provincia, creciente } \\
\text { depredación de bosques, especies vegetales y fauna nativas, ausencia de tratamiento de aguas y } \\
\text { residuos sólidos. }\end{array}$} \\
\hline $\begin{array}{l}\text { dación tributa- } \\
\text { promoción del } \\
\text { ollo económico }\end{array}$ & \multicolumn{2}{|c|}{$\begin{array}{l}\text { Baja recaudación tributaria e incremento de conflictos entre funcionarios y ciudadanía sobre el marco } \\
\text { normativo tributario. } \\
\text { Desatención a Pymes de producción artesanal y servicios turísticos, creciente tasa de desempleo } \\
\text { entre PEA menos de } 20 \text { años. } \\
\text { Desatención a la producción agrícola (infraestructura de riego, capacidades y captación de nuevos } \\
\text { mercados). } \\
\text { En el campo de la gestión tributaria, de forma unánime, opinaron que debería mejorar ya que es el } \\
\text { pilar de los ingresos directamente recaudados, necesarios para una mejor gestión de la institución. } \\
\text { En cuanto a la reanudación tributaria el } 100 \% \text { de funcionarios coinciden que es importante para la } \\
\text { gestión (Fig. 6). }\end{array}$} \\
\hline \multicolumn{2}{|c|}{$\begin{array}{l}\text { Gestión Institucional } \\
\text { les de la gestión deberían ser capacitados? }\end{array}$} & \\
\hline & & \\
\hline
\end{tabular}

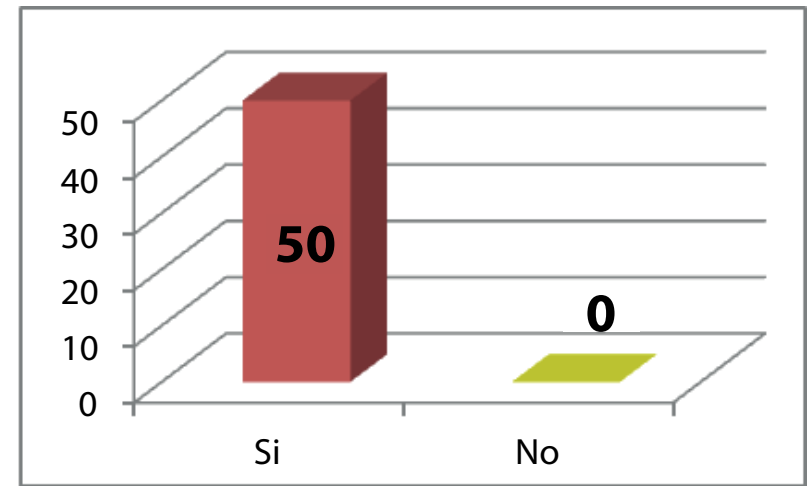

Fig. N. ${ }^{\circ} 1$

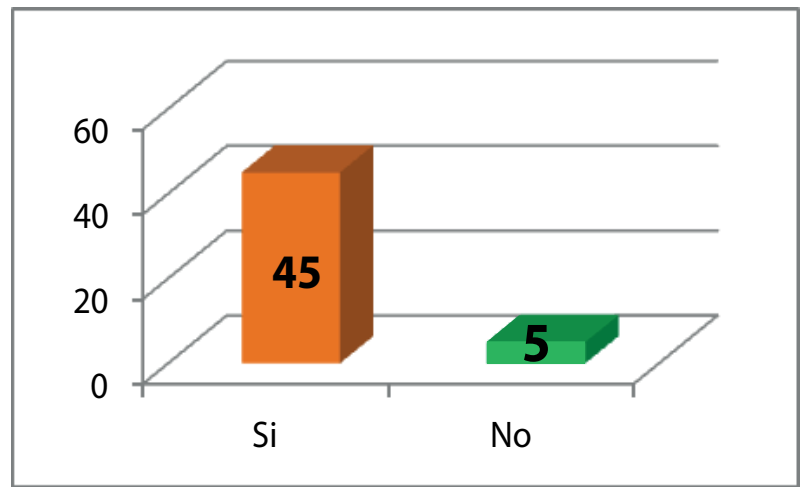

Fig. N. ${ }^{\circ} 2$

\begin{tabular}{|c|cc|}
\hline \multicolumn{2}{|c|}{ Infraestructura vial y desarrollo urbano } \\
¿Es necesario establecer planes para el desarrollo vial y \\
urbano?
\end{tabular}




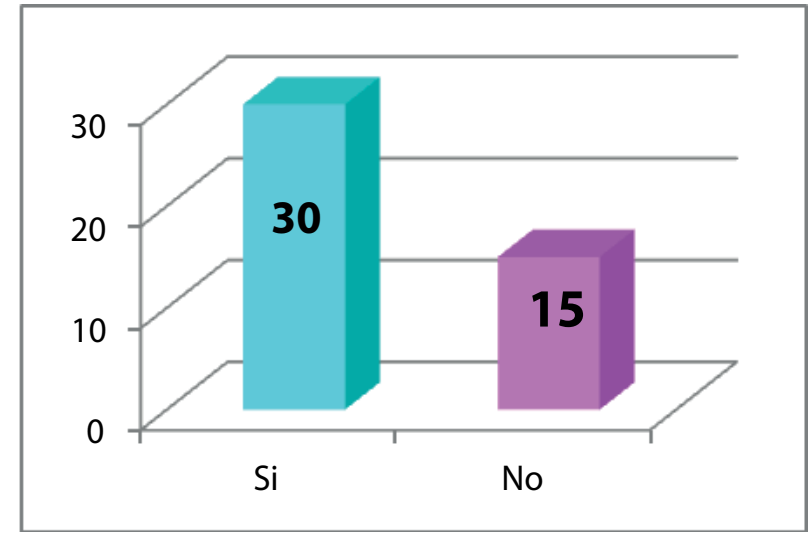

Fig. $\mathrm{N}^{\circ} 3$

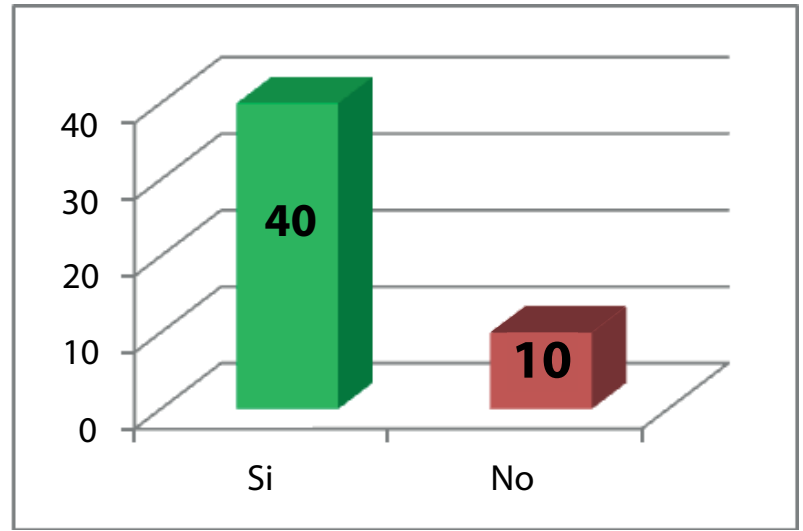

Fig. N. ${ }^{\circ} 4$
Gestión de los recursos naturales y el cuidado del ambiente

1. ¿Es necesario elaborar un plan estratégico de gestión Sí: 50 ambiental?

No: 0
Gestión tributaria y promoción del desarrollo económico local

2. ¿Considera Ud. que la gestión tributaria y promoción es importante para la gestión? Sí: 50
No: 0

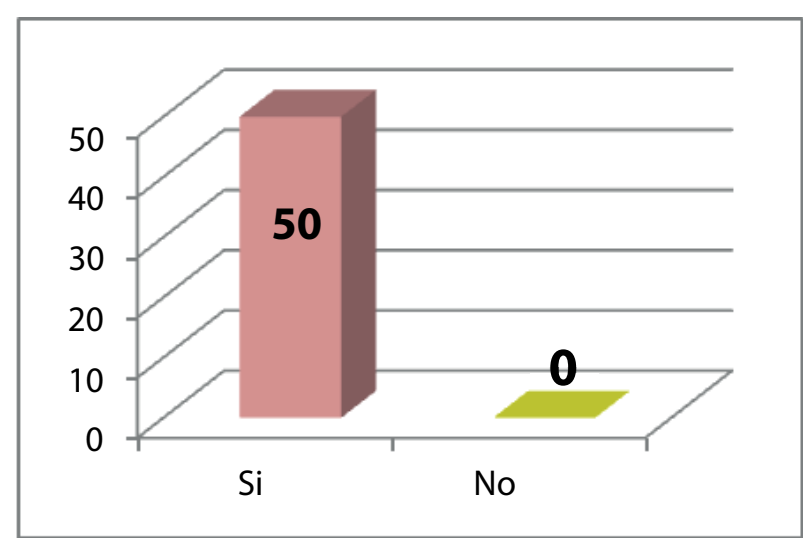

Fig. $\mathrm{N}^{\circ} 5$

Los indicadores relacionados con el IDH tabla N ${ }^{\circ} 1$ y Minsa (Tabla N. ${ }^{\circ} 2$ ), línea de base para la gestión evaluada 2007-2010, reflejan elevadas carencias y el estudio no encontró antecedentes en los instrumentos de gestión del Órgano de Gobierno Municipal como resultado de metas, objetivos o políticas institucionales. La alcaldía y el concejo municipal tienen las atribuciones y autonomía señaladas por "LOM" para ejercer gobierno, promoviendo la satisfacción de las necesidades de la población y el desarrollo de su ámbito (3). Las instancias de representación promotora del desarrollo integral sostenible y prestadora de servicios públicos, dicho de otro modo, razón de ser del municipio, están planteadas pensando en el protagonismo del alcalde y en la escasa producción normativa y fiscalización de la comisión de regidores.

Las desviaciones y limitaciones deducidas de los puntos a, b, c, d, e, f y g de La tabla N. ${ }^{\circ} 3$, aprobado y programado en los objetivos institucionales: "Función de gobierno municipal" y las cifras obteni-

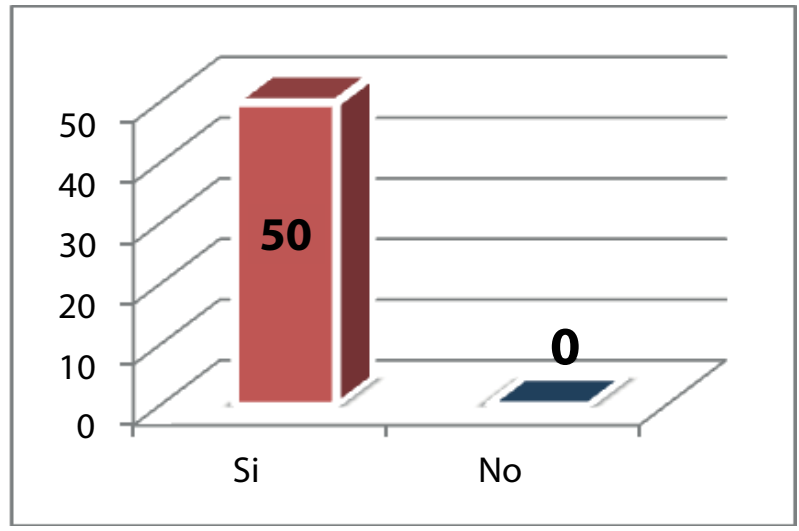

Fig. N. ${ }^{\circ} 6$

das, es el cumplimiento de los desafíos básicos que responden los órganos de dirección encargados de dar cumplimiento a lo emanado del gobierno municipal. La "Función técnica" (8) está en la facultad de proveer realineamientos en las metas, objetivos y políticas del gobierno institucional en el marco de las diversas atribuciones de poder que la ley municipalista otorga al alcalde. La "Función ejecutiva"(3) muestra incumplimiento de objetivos direccionados por el estilo de gestión realizada.

Reflexionando en los puntos h, i, j, k, I, m, n, o, p y q de la tabla $N .{ }^{\circ} 3$. Aspectos como educación, seguridad ciudadana, salud, electrificación o saneamiento, racionalización y simplificación de los procedimientos administrativos y la optimización de los recursos humanos no muestran cifras esperanzadoras a pesar de la capacidad de gestión independiente facultada para decidir y ordenar sus funciones y competencias exclusivas (autonormarse) a través de autonomía (3): política, emitir normas con naturaleza de ley. Autonomía económica, generar 
sus propios recursos y disponer de los que tiene y administrativa, realizar actos administrativos en la organización y estructura municipal a través de los componentes de apoyo, línea y asesoramiento para liderar la gestión de desarrollo integral sostenible de su ámbito y mejorar la calidad de vida de la población, de aquellos que se muestran en pobreza extrema.

Los hallazgos en los puntos $r, \mathrm{~s}, \mathrm{t}, \mathrm{u}, \mathrm{v}, \mathrm{w}, \mathrm{x}$ í en la tabla $\mathrm{N} .{ }^{\circ} 3$, muestran contradicciones para el cumplimiento eficaz como instancia prestadora de servicios públicos, puesto que los de interés generadores de conflictos sociales que alteran el bienestar individual o colectivo no muestran atención preferente, soslayando el mandato otorgado y recibido en las ánforas para conducir y permitir a la población intervenir en los asuntos de gestión para garantizar eficiencia y transparencia.

La información de la tabla $\mathrm{N}^{\circ} 3$ urge de las facultades de los Órganos de Gobierno y Dirección en el marco de la descentralización y la Ley de Municipalidades. Dosificar el modelo tradicional gestión aplicado por la mayoría de gobiernos locales a través de estilos de administración centrando todo el esfuerzo en el bienestar del usuario; se puede objetar ante un Modelo de Eficacia (8), y se hace necesaria una definición político-estratégica. Sin embargo, se trata de ámbitos de trabajos distintos, no necesariamente discrepantes. En otras palabras, tanto si inclinamos la gestión municipal hacia el turismo incentivando así el desarrollo de promotores locales, como sí lo dirigimos a la agro exportación con capital extranjero -lo cual puede implicar ideologías políticas en pugna-, las cifras halladas cambiarían si optimizamos recursos para promover el desarrollo socioeconómico y elevar el nivel de vida de los vecinos.

\section{CONCLUSIONES}

1. Los Órganos de Gobierno y Dirección municipal del siglo $X X$, con gestores públicos apremiados por ordenar a sus inferiores a cumplir con los preceptos de la contraloría, es decir vigilar que los funcionarios públicos satisfagan normas administrativas y de probidad. Sin lugar para la discrecionalidad, el modelo organizacional tradicional, burocrático, jerárquico y predominante (8) ya no resuelve el cambio de las necesidades de la población de esta compleja época; que altera los volúmenes de carga de trabajo y expansión de servicios, consecuencias que deben solucionar la municipalidad.

2. Las municipalidades necesitan liberarse del exceso burocrático, así como modernizarse frente a los organismos del gobierno central. Se requiere maximizar la capacidad del gobierno local para motivar la participación de los vecinos, incluyendo las redes sociales que se producen en una comunidad, con la fiscalización y el control de los servicios como paradigma de gestión, liderando el desarrollo local en las diversas modalidades de servicios que desarrollen, satisfaciendo las necesidades de gobierno y los servicios locales.

3. El Modelo Europeo de Excelencia Empresarial, EFQM, elegido para su aplicación en el Municipio de Otuzco, comprende dos aspectos fundamentales: un conjunto de criterios de excelencia de gestion que abarcan todas las áreas del funcionamiento de la organización, así como un conjunto de reglas para evaluar el comportamiento de la organización en cada criterio.

4. Es a partir de este modelo que se puede enjuiciar objetivamente una gestión para reducir gastos administrativos (2), eliminando cargas innecesarias de trabajo, evitando el desperdicio de mobiliario y servicios, desconcentrando y estableciendo responsabilidades en cada nivel, evitando la innecesaria concentración y los "cuellos de botella". Disminuir el presupuesto de funcionamiento, eliminando las instancias innecesarias, delimitando campos y duplicación de funciones, exigiendo cero desperdicios de materiales y esfuerzos congelando los niveles de decisión inapropiados.

5. La cadena de mando, agiliza la estructura y estableciendo el número adecuado de jefes que deben reportar al Gobierno y Dirección municipal. Optimizar los recursos (2), reforzando los órganos de línea, apoyo y asesoramiento, mediante una estructura simple desburocratizada y funcional para efectuar nuevas actividades (diseñando nuevos órganos para cumplir nuevas funciones); proporcionando mejores medios de coordinación (estableciendo sistemas de consulta, intercambio de información, etc.). Dignificar la vida rural (2), donde los nuestros niños sufren frío, desnutrición y enfermedades endémicas por falta de servicios sanitarios, de salud y nutrición adecuada oportuna y eficiente. La ausencia de agua potable y alcantarillado genera diarreas lo cual genere la desnutrición, y en consecuencia, anemia, TBC y otras dolencias; donde la niñez y adolescencia sean el verdadero presente y futuro, aumentando el presupuesto de inversión, priorizando la inversión en los servicios de salud, seguridad ciudadana, participación activa de la juventud y las Juntas Vecinales. Uniendose así el municipio con el sector privado y los agentes locales para explotar el potencial endógeno existente, y brindar servicios eficientes al vecindario en cantidad y calidad en un desarrollo equilibrado del Municipio de Otuzco.

6. Se puede iniciar un trabajo dentro del municipio con participación de las autoridades, sectores 
gubernamentales, instituciones involucradas en la temática y la comunidad en general, buscando generar espacios de consenso en torno a estrategias sostenibles, a fin de alcanzar adecuados niveles de vida de la población otuzcana. El trabajo emprendido y para ello contribuye de manera efectiva al proceso de mejora continua, equitativa e inclusiva, precisa de una dirección integral y un manejo de resultados de excelencia con elevado impacto en la calidad de vida de la ciudadanía. Es necesario centrar todos los esfuerzos para revertir el reporte de la Tabla N. ${ }^{\circ} 3$, redefiniendo variables acotadas en función de los Órganos de Gobierno y Dirección Municipal asumiendo su riesgo financiero y político por sus decisiones ante la comunidad. Así mismo, urge reformular la Ley de Bases de la descentralización (Ley $\mathrm{N}^{\circ}{ }^{\circ}$ 27867), debido a las limitaciones que apreciamos en el proceso de descentralización a fin de lograr una sociedad más justa e inclusiva.

7. La tarea retrasada de la municipalidad es modificar su finalidad y objetivos fundamentales con nuevos campos funcionales mediante un instrumento de gestión diferente, incorporar metodologías de participación ciudadana a la planificación cuando se gestan los programas y grandes decisiones evitando costos de imagen pública difíciles de aminorar. Las competencias actuales y la legislación municipal vigente apertura a los cambios requeridos definiendo las variables mensurables que sirvan de referencia para profesionalizar la gestión y aglutinar a los empresarios informales que se hallan en pobreza extrema. Donde solo es posible actuar localmente a través de sistemas de articulación empresarial y que el municipio por su proximidad al ciudadano y el conocimiento de su cultura debería aprovechar.

8. La mayor parte de los modelos están inspirados en el Total Quality Management (TQM)(1) que busca el mejoramiento continuo de la organización como un todo, satisfacción de clientes y stakeholders; los de la excelencia se basan en el Malcolm Baldrige y se puede decir que quizá es el más completo pues incorpora una mayor cantidad de criterios; sin embargo, el más específico para la prestación de servicios municipales y es el modelo EFQM(5) a través de 32 subcriterios. Tras un análisis metódico, se concluyó que, en la Municipalidad de Otuzco, existen razones valederas y suficientes para implementarse un Plan Estratégico consensuado que parta de un estudio científico efectivo de la realidad, este Plan deberá implementar un Modelo de Gestión de Excelen- cia EFMQ (\%)(5) de la fundación europea; lo cual permitiría ser más eficiente y eficaz en el desarrollo institucional y ser sostenido en el tiempo, considerando la experiencia de los ayuntamientos españoles. Cualquiera sea la política estratégica a seguir, el Modelo EFQM aportará indicadores y sistemas con funcionarios centrados en organizar recursos que frecuentemente pertenecen a otros, para producir valor público y no en gerenciar personas y programas cuyos resultados mensurables puedan ser criticados objetivamente y mantenidos con independencia de la orientación política de los gestores ediles.

\section{REFERENCIAS BIBLIOGRÁFICAS}

[1] Aguilar, I. (2008). Gestión de la calidad en los municipios mexicanos. Un estudio de caso: Ecatepec de Morelos, Estado de México, 2003-2006. Recuperado de http://www.clad.org/siare_isis/fulltext/0059402.pdf (visitado el 19-03-2011)

[2] Casermeiro, M. (2008). La experiencia en gestión de la calidad en la provincia de Salta. Revista de la Red de Expertos Iberoamericanos en Calidad en la Administración Pública, 2, 14-17. Recuperado dehttp://www.aeval.es/comun/pdf/DAD_ Nx_2_1S_08.pdf

[3] Contraloría general de la República del Perú Contraloría General de la República es el órgano superior del Sistema Nacional de Control, cautela el uso eficiente, eficaz y económico de los recursos www. contraloria.gob.pe/ (Visitado 10- 02- 2011).

[4] Deming, E. (2000) "Out of crisis" The Mit Press: Cambridge.

[5] Efqm. Fundación Europea para la Gestión de la Calidad. www.efqm.org. (Visitado 22-04-2011).

[6] Fundación Iberoamericana para la gestión de la calidad (FUNDIBEQ). www.fundibeq.org. (Visitado 12-01-2011).

[7] Hernández, Roberto y otros. (2000). Metodología de la Investigación $2^{a}$ Edición; Editorial Me GrawHill: México.

[8] Juanes, B. y Blanco, J. (2001). El gato de Alicia. Modelos de calidad en la administración pública. Madrid: Galgano España y Díaz de Santos.

[9] Sierra, Roberto. (1998). "Tesis Doctorales y Trabajos de Investigación Científica”; Edición $4^{\text {ta; }}$ Editorial Trillas:México.

[10] The Baldrige Model. www.baldrige.com/. (Visitado 10-01- 2011). 